\title{
A STRONG HOMOTOPY AXIOM FOR ALEXANDER COHOMOLOGY
}

\author{
KERMIT SIGMON
}

\begin{abstract}
It is shown that the following form of the homotopy axiom holds for Alexander-Cech cohomology. Suppose that $X$ and $Y$ are any spaces, that $T$ is a compact, connected space, and that $G$ is an abelian group which either admits the structure of a compact topological group or is the additive group of a finite-dimensional vector space. Then for any continuous function $F: X \times T \rightarrow Y$, one has $F_{r}^{*}=F_{s}^{*}: H^{*}(Y ; G) \rightarrow H^{*}(X ; G)$ for all $r, s \in T$, where $F_{t}: X \rightarrow$ $Y$ is defined by $F_{t}(x)=F(x, t)$.
\end{abstract}

1. Introduction. The purpose of this paper is to prove the following theorem.

THEOREM. Suppose that $X$ and $Y$ are any spaces, that $T$ is a compact, connected space, and that $G$ is a compact abelian topological group or a finite-dimensional vector space. Then for any continuous function $F: X \times$ $T \rightarrow Y$, one has

$$
F_{r}^{*}=F_{s}^{*}: H^{*}(Y ; G) \rightarrow H^{*}(X ; G)
$$

for all $r, s$ in $T$, where $F_{t}: X \rightarrow Y$ is defined by $F_{t}(x)=F(x, t)$.

While we prove the theorem for Alexander (more accurately, Alexander-Kolmogoroff) cohomology, it also holds for Cech cohomology since the two are naturally equivalent [2]. The theorem also holds for topological pairs, as a straightforward modification of our proof will show. We restrict our attention to the single space case for simplicity of presentation.

In case $T$ is an arc, the theorem is well known (no restriction on $G$ ), following either from its truth in the Cech theory [1] and the equivalence of the Alexander and Čech theories [2] or from a direct proof for the Alexander theory ([6] or [5, p. 314]). On the other hand, it is known that if $X$ is compact, the theorem holds with $T$ any connected space (again, no rectriction on $G$ ) as an easy generalization of the proof in $[4, \mathrm{p}, 416]$ shows. The freedom of choosing the parameter space to be continuum, which is allowed by taking $X$ to be compact, has proved very useful in the study

Received by the editors May 20, 1970.

AMS 1970 subject classifications. Primary 55B05, 55B40; Secondary 22A15. 
of topological semigroups. Our theorem retains this freedom of choice of parameter space while removing the compactness restriction on $X$, provided one chooses an appropriate coefficient group. It is a trivial observation, of course, that one does not really need to assume $T$ to be compact, only that each pair of points of $T$ is contained in some compact, connected subset of $T$ (e.g., $T$ locally compact, locally connected, connected). For further discussion of the problem the reader is referred to the review by K. Hofmann in Mathematical Reviews 32 (1966), p. 514.

The author is indebted to his colleague Philip Bacon for many helpful comments.

2. Preliminaries. In this section we collect several preliminary results we will need later.

If $X$ is a set and $U$ is a collection of sets, we denote by $X_{\mathcal{U}}$ the simplicial complex whose simplexes are the finite nonempty subsets of $X$ which are contained in some member of $\mathcal{U}$.

Proposition 1. For each space $X$ and open cover $U$ of $X$ there is a homomorphism $i_{น}: H^{*}\left(X_{น}\right) \rightarrow H^{*}(X)$ such that $\left\{H^{*}(X), i_{น}\right\}$ is a direct

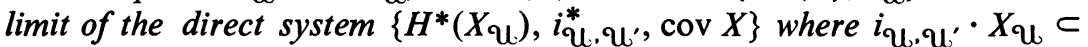
$X_{\mathcal{U}^{\prime}}\left(\mathcal{U}\right.$ refines $\left.{ }^{\prime}\right)$. Furthermore, if $f: X \rightarrow Y$ is a map and if $\mathcal{U}$ and $v$ are covers of $X$ and $Y$, respectively, such that $\{f(U) \mid U \in \mathcal{U}\}$ refines $V$, then the diagram

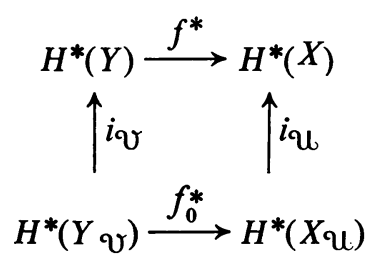

commutes, where $f_{0}: X_{\mathcal{U}} \rightarrow Y_{\vartheta}$ is the simplicial map induced by $f$.

For a proof of this proposition, see [5, p. 312].

Although the functor $H^{*}$ does not, in general, commute with inverse limits, the following proposition holds. Its proof is an appropriate modification of that given for the special case of exact sequences in [3, p. 226].

Proposition 2. On either the category of cochain complexes over compact abelian topological groups or the category of cochain complexes over finite-dimensional vector spaces over a fixed field, the functor $H^{*}$ commutes with inverse limits.

For a simplicial complex $K, C^{p}(K ; G)$ is defined as $\left\{\varphi \mid \varphi: K_{p} \rightarrow G\right\}$, where $K_{p}=\left\{\left(\chi_{0}, \cdots, \chi_{p}\right) \mid\left\{\chi_{0}, \cdots, \chi_{p}\right\} \in K\right\} . C^{p}(K ; G)$ is then just a 
cartesian product of copies of $G$. Hence, we have the following propositions. The proof of the continuity, resp. linearity, of $\delta$ and $f^{\#}$ is straightforward.

Proposition 3. If $G$ is a compact abelian topological group and $K$ is a simplicial complex, then $C^{p}(K ; G)$ is a compact abelian topological group. Furthermore, $\delta: C^{p}(K ; G) \rightarrow C^{p+1}(K ; G)$ and, for a simplicial map $f: K \rightarrow K^{\prime}, f^{\#}: C^{p}\left(K^{\prime} ; G\right) \rightarrow C^{p}(K ; G)$ are continuous.

Proposition 3'. If $G$ is a finite-dimensional vector space and $K$ is a finite simplicial complex, then $C^{p}(K ; G)$ is a finite-dimensional vector space. Furthermore, $\delta: C^{p}(K ; G) \rightarrow C^{p+1}(K ; G)$ and, for a simplicial map $f: K \rightarrow K^{\prime}, f^{\#}: C^{p}\left(K^{\prime} ; G\right) \rightarrow C^{p}(K ; G)$ are linear transformations.

The proof of the following proposition is simple and hence omitted.

Proposition 4. Suppose $K$ is a simplicial complex and $\mathrm{C}$ is a collection of subcomplexes of $K$ satisfying

(i) $\cup \mathrm{C}=K$, and

(ii) if $A, B \in \mathrm{C}$, then $A \cup B$ is contained in some member of $\mathrm{C}$.

Then, for any abelian group $G,\left\{C^{*}(K ; G), p_{A}^{\#}\right\}$ is an inverse limit of the inverse system $\left\{C^{*}(A ; G), i_{A, B}^{\#}, \mathcal{C}\right\}$, where $i_{A, B}: A \subset B$ and $p_{A}: A \subset K$.

3. Proof of the Theorem. We prove the following Lemma, which is easily seen to be equivalent to the theorem.

LEMMA. Suppose that $X$ and $T$ are spaces with $T$ compact and connected and that $G$ is either a compact abelian topological group or a finite-dimensional vector space. If $\lambda_{t}: X \rightarrow X \times T$ is defined for each $t$ in $T$ by $\lambda_{t}(x)=$ $(x, t)$, then $\lambda_{r}^{*}=\lambda_{s}^{*}: H^{*}(X \times T ; G) \rightarrow H^{*}(X ; G)$ for all $r, s$ in $T$.

Proof. Let $g \in H^{p}(X \times T)$. We wish to show that $\lambda_{r}^{*}(g)=\lambda_{s}^{*}(g)$. From Proposition 1 we have that there is an open cover $\mathcal{V}$ of $X \times T$ and $g_{\vartheta} \in H^{p}((H \times T))_{\mho}$ such that $i_{\vartheta}\left(g_{\vartheta}\right)=g$, where $i_{\vartheta}$ is the canonical injection. Using the compactness of $T$, we get for each $x \in X$ an open set $U_{x}$ about $x$ and an open cover $\mathscr{D}_{U_{x}}$ of $T$ such that $\left\{U_{x} \times D \mid D \in \mathscr{D}_{U_{x}}\right\}$ refines $\mathcal{V}$. We let $U$ denote the open cover $\left\{U_{x} \mid x \in X\right\}$ of $X$ and let $\mathcal{K}$ denote the collection of all ordered pairs $(\mathcal{C}, F)$ where $\mathcal{C}$ is a finite subcollection of $\mathcal{U}$ and $F$ is a finite subset of $X$ with $F \subset \cup \mathcal{G}$. By defining $\left(\mathcal{C}_{1}, F_{1}\right) \leqq\left(\mathcal{C}_{2}, F_{2}\right)$ iff $\mathcal{C}_{1} \subset \mathfrak{C}_{2}$ and $F_{1} \subset F_{2}, \mathcal{K}$ becomes a directed set. It is clear that, for each $t$ in $T, \lambda_{t}$ induces simplicial maps $\lambda_{t}^{\mathcal{U}}: X_{\mathcal{U}} \rightarrow$ $(X \times T)_{\mathcal{v}}$ and $\lambda_{t}^{K}: F_{\mathcal{G}} \rightarrow(X \times T)_{\mathcal{v}}, K=(\mathcal{G}, F) \in \mathcal{K}$.

We show first that, for each $K=(\mathcal{C}, F) \in \mathcal{K}$, the chain maps $\left(\lambda_{r}^{K}\right)^{\#}$, $\left(\lambda_{s}^{K}\right)^{\#}: C^{*}\left((X \times T)_{\mathcal{U}}\right) \rightarrow C^{*}\left(F_{\mathcal{C}}\right)$ are chain homotopic. Let $K=(\mathcal{G}, F) \in \mathcal{K}$ 
with $\mathfrak{C}=\left\{U_{1}, \cdots, U_{n}\right\}$ and let $\mathfrak{D}$ be a common open refinement of $\mathfrak{D}_{U_{1}}, \cdots, \mathfrak{D}_{U_{n}}$ covering $T$. Since $T$ is connected there exist $D_{1}, \cdots$, $D_{k} \in \mathcal{D}$ and $p_{0}, \cdots, p_{k}$ such that $r=p_{0} \in D_{1}, s=p_{k} \in D_{k}$ and $p_{i} \in D_{i} \cap$ $D_{i+1}, 1 \leqq i \leqq k-1$. For each $i=1, \cdots, k, \lambda_{p_{i}}^{K}$ and $\lambda_{p_{i}-1}^{K}: F_{\mathcal{\zeta}} \rightarrow$ $(X \times T)_{\text {v }}$ are contiguous since, for each $i=1, \cdots, n, \lambda_{p_{i}}^{K}\left(U_{i}\right)=$ $U_{i} \times\left\{p_{i}\right\} \subset U_{i} \times D_{i}$ and $\lambda_{p_{i}-1}^{K}\left(U_{i}\right)=U_{i} \times\left\{p_{i-1}\right\} \subset U_{i} \times D_{i}$ with $U_{i} \times$ $D_{i} \subset U_{i} \times D \subset V$ for some $D \in \mathscr{D}_{U_{i}}$ and $V \in \mathcal{V}$. It follows that, for each $i=1, \cdots, k,\left(\lambda_{p_{i}}^{K}\right)^{\#}$ and $\left(\lambda_{p_{i}-1}^{K}\right)^{\#^{*}}$ are chain homotopic and hence so are $\left(\lambda_{r}^{K}\right)^{\#}$ and $\left(\lambda_{s}^{K}\right)^{\#}$.

We now complete the proof of the Lemma. It is immediate from Proposition 4 that $\left\{C^{*}\left(X_{U}\right), i_{K}^{\#}\right\}$ is an inverse limit of the inverse system $\left\{C^{*}\left(F_{\mathcal{C}}\right), i_{K, K^{\prime}}^{\#}, \mathcal{K}\right\}$ where $i_{K}: F_{\mathcal{C}} \subset X_{\mathcal{U}}$ and $i_{K, K^{\prime}}: F_{\mathcal{C}} \subset F_{\mathcal{G}^{\prime}}^{\prime}, K \leqq K^{\prime}$, $K=(\mathscr{C}, F), K^{\prime}=\left(\mathfrak{C}^{\prime}, F^{\prime}\right)$. From Propositions 2,3 , and $3^{\prime}$ we then have that $\left\{H^{p}\left(X_{\mathcal{U}}\right), i_{K}^{*}\right\}$ is an inverse limit of the system $\left\{H^{p}\left(F_{\mathcal{C}}\right), i_{K, K^{\prime}}^{*}, \mathcal{K}\right\}$. For each $t \in T$ and $K, K^{\prime} \in \mathcal{K}, K \leqq K^{\prime}$, the diagrams

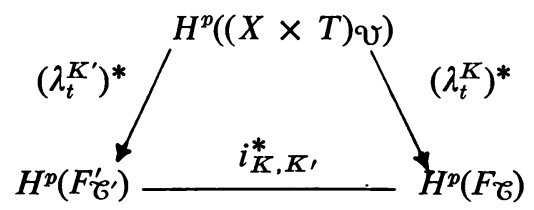

and

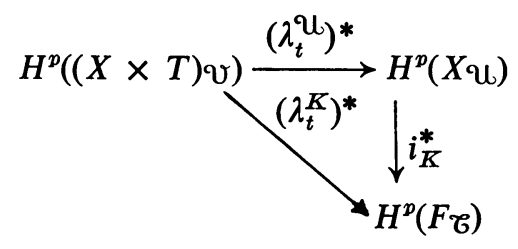

commute. But $\left(\lambda_{r}^{K}\right)^{*}=\left(\lambda_{s}^{K}\right)^{*}$ for each $K \in \pi$ since $\left(\lambda_{r}^{K}\right)^{\#}$ and $\left(\lambda_{s}^{K}\right)^{\#}$ are chain homotopic, so from the uniqueness condition in the characteristic property of inverse limits one has $\left(\lambda_{r}^{\mathcal{U}}\right)^{*}=\left(\lambda_{s}^{\mathcal{U}}\right)^{*}$. From this and the commutativity of

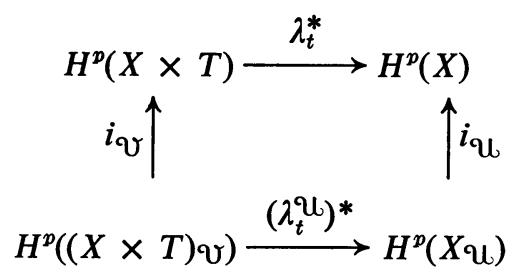

(Proposition 1) one obtains $\lambda_{r}^{*}(g)=\lambda_{s}^{*}(g)$, completing the proof. 


\section{REFERENCES}

1. C. H. Dowker, Cech cohomology theory and the axioms, Ann. of Math. (2) 51 (1950), 278-292. MR 11, 450.

2. - , Homology groups of relations, Ann. of Math. (2) 56 (1952), 84-95. MR 13, 967.

3. S. Eilenberg and N. Steenrod, Foundations of algebraic topology, Princeton Univ. Press, Princeton, N.J., 1952. MR 14, 398.

4. E. H. Spanier, Cohomology theory for general spaces, Ann. of Math. (2) 49 (1948), 407-427. MR 9, 523.

5. —, Algebraic topology, McGraw-Hill, New York, 1966. MR 35 \#1007.

6. P. L. Sperry, The homotopy axiom for Alexander theory, J. London Math. Soc. 41 (1966), 97-100. MR 32 \#3044.

Department of Mathematics, University of Florida, Gainesville, Florida 32603 